differ profoundly according to the intensity of Feulgen's reaction. The proximal ends of the autosomes continue to unite with the heterochromatin region of the $X$-chromosome in the same way as they united formerly with the chromocentre.

When the chromosome bands begin to form, the heterochromatin region extends still more and the small number of chromomeres of early stages disintegrate lengthwise into a greater number. When the genonemata become double, the number of chromomeres in the row is also doubled in both euchromatin and heterochromatin chromosome regions. Thus a typical picture of the inert region of the $X$-chromosome of $D$. funebris in the salivary gland nuclei of large larvæ is obtained.

When the bands in the salivary gland chromosomes of $D$. melanogaster are formed, the round chromocentre of a resting nucleus divides into parts belonging to separate chromosomes. These chromosome parts divide into chromomeres later on.

In $D$. repleta the double-armed $X$-chromosome is represented only by one banded chromosome in the salivary gland nuclei. This can be explained only by the fact that the second inert arm forms the chromocentre, which in the large salivary gland nuclei does not differ externally from the chromocentre of the resting nuclei. In other words, this arm retains the same compact condition as in the resting nucleus.

In the large salivary gland nuclei of $D$. virilis and $D$. robusta, the chromocentre of a resting nucleus is retained as in $D$. repleta, but is decreased in dimensions. This can be explained by the fact that in young larvæ small parts separate from the chromocentre and disintegrate into chromomeres. Later on, the number of chromomeres doubles exactly in the same way as in the euchromatin chromosome regions. The remaining part of the chromocentre represents a fusion of small parts of heterochromatin, proximal ends of all the chromosomes in $D$. robusta and in $D$. virilis all the heterochromatin of chromosome III in addition.

The differences of the structure of the point of union of the proximal ends of all the chromosomes in the salivary gland nuclei of large larvæ of different species of Drosophila can thus be explained, whether the whole or a section of the chromocentre of a resting nuclei is transformed into the inert chromosome regions, that is, obtains a chromomere structure.

The thread leading from the chromocentre to the nucleus is formed during the transition from telophase to the resting nucleus. Its nature has so far not been made clear.

S. Frolova.

Institute of Experimental Biology, Moscow. June 17.

${ }^{1}$ Frolova, NATURE, 141, 1015 (1938).

2 Neitz, Biol. Zol., 54, 11/12 (1934),

${ }^{3}$ Heitz, Z. Zellf. u. micr. Anat., 19 (1933).

\section{Chromosome Numbers in Cimex}

A sub-species of Cimex, phenotypically intermediate between $C$. lectularius and $C$. columbarius and obtained from laboratory white rats, has been found to comprise two forms differing in the number of chromosomes. In most cases the haploid number of autosomes is twenty-four, but in occasional specimens it is sixteen. No variation in phenotype, correlated with this change in number, has been observed, nor is the normal course of meiosis affected.

This suggests that eight autosomes in this subspecies either represent a duplicate set the functions of which can be performed by the remainder of the complement, or are genetically jnert.

All $C$. lectularius material so far examined has yielded a haploid autosome count of twenty. Since these three karyotypes form a series having chromosome numbers which are multiples of four, there is reason to suppose that $(a)$ the genus is polyploid in origin, (b) the stem number in Cimex is four and not six as was suggested by Slack ${ }^{1}$ and Vandel $^{2}$ for the Heteroptera.

$$
\begin{aligned}
& \text { University, } \\
& \text { Glasgow. } \\
& \text { June } 28 .
\end{aligned}
$$

${ }^{1}$ Slack, H. D., British Association, Nottingham (1937) (unpublished). 2 Vandel, A., Proc. Zool. Soc., A, 107, 519 (1937).

\section{Time Sequence of Crossing-Over}

MATHER $^{1}$ has answered the challenge of Charles ${ }^{2}$ regarding the time sequence of crossing-over. Like Charles, I feel sceptical of Mather's evidence ${ }^{3}$ that crossing-over begins near the centromere.

Mather suggests that the experiments on the effect of age, temperature and inversions in Drosophila show the differential effect between the centromere and the ends of the chromosome to be expected on his hypothesis. Before he can use this evidence as a support for his hypothesis, he must first show that the genetically unsplit region near the centromere behaves similarly to the ends of the chromosomes in all other respects than crossing-over. It is perfectly reasonable to suppose that the genetically unsplit region and its neighbourhood react quite differently to external influences. Indeed there is much evidence for this, but it is not necessary to assume, as Mather does, that the observed changes in linkage are due to the fact that the first formed chiasma is proximal to the centromere. If, for example, an increase in temperature influences the distance at which the chromosome is genetically split at the time of crossingover, the changes in linkage values will be observed no matter where crossing-over started on the chromosome.

The precocious splitting in unpaired parts of trivalent or univalent chromosomes provides more factual evidence than that adduced by Mather. The papers by Charles, Schweitzer and Mather are most useful in suggesting novel modes of attack; but indicate the dangers of jumping to conclusions, however reasonable, which are derived from the cytological or statistical methods at present favoured by many.

Until the mechanics of chromosome pairing are understood, it is difficult to utilize the data of pairing in structurally changed forms in the way Mather has done. A juxtaposition of the centromeres at early meiotic prophase as seen in salivary glands would account for the behaviour of the heterozygote of the Delta 49 inversion, but without factual evidence such a suggestion is as useless as Mather's. It is possible that Mather is correct in his assumption regarding this theory, but more genetical evidence is required before acceptance is possible.

Botanical Department,

F. W. Sansome.

University of Manchester, Manchester, 13. July 25.

1 Mather, K., NATURE, 142, 157-158 (1938).

2 Charles, D. R., J. Genet, 36, 103-26 (1938).

${ }^{3}$ Mather, K., J. Genet., 33, 207-35 (1936). 